\title{
Water-Dispersible Multiwalled Carbon Nanotubes Obtained from Citric-Acid-Assisted Oxygen Plasma Functionalization
}

\author{
Mohammad Jellur Rahman ${ }^{1}$ and Tetsu Mieno ${ }^{1,2}$ \\ ${ }^{1}$ Graduate School of Science \& Technology, Shizuoka University, Shizuoka 422-8529, Japan \\ ${ }^{2}$ Department of Physics, Shizuoka University, Shizuoka 422-8529, Japan \\ Correspondence should be addressed to Tetsu Mieno; sptmien@ipc.shizuoka.ac.jp
}

Received 18 April 2014; Accepted 3 June 2014; Published 18 June 2014

Academic Editor: Shota Kuwahara

Copyright (C) 2014 M. J. Rahman and T. Mieno. This is an open access article distributed under the Creative Commons Attribution License, which permits unrestricted use, distribution, and reproduction in any medium, provided the original work is properly cited.

A new and safe method has been developed to functionalize multiwalled carbon nanotubes (MWCNTs) with fewer surface defects, which significantly increases their dispersibility in water. MWCNTs are pretreated in pure ethanol by a supersonic homogenizer. Then, the mixture is dried and soaked in weak citric acid solution. Finally, the MWCNTs in the citric acid solution are treated with radio frequency $(13.56 \mathrm{MHz})$ oxygen plasma. As a result, many carboxyl functional groups are attached onto the MWCNT surfaces and stable dispersion of the MWCNTs in water is obtained. The treatment conditions are optimized in this study.

\section{Introduction}

Water-dispersible carbon nanotubes (CNTs) have recently become of great importance owing to their promising chemical and physical properties. The most obvious benefits of these water-dispersible CNTs are their value in biochemistry and biomedical engineering, electronic technologies of films, plastic materials, and polymer composites $[1,2]$. However, these applications are compromised by their poor dispersibility in water, because substantial van der Waals attractive forces between the CNTs aggregate them in solvents [3]. The most common method of improving their dispersibility in water is to functionalize their surfaces using hydrophilic oxygen-containing groups [4]. This is achieved by oxidizing CNTs by strong acids, such as refluxing in a mixture of sulfuric acid and nitric acid $[5,6]$, "piranha" solution (sulfuric acid-hydrogen peroxide) [7], boiling in nitric acid [8], or treating with oxidative gases, such as ozone $[4,9]$. However, treatment under such harsh conditions clearly deviates from green chemistry and results in the opening of the tube tips [5], shortening of the tubes [7], and fragmentation of the sidewalls [8]. Therefore, their stability decreases and their integral properties change. Also, the acidic treatment often requires a long processing time and produces a large amount of waste. To overcome these problems, alternative safer and easier functionalization methods should be considered.

In these respects, plasma processing is an advantageous technique when it is used in conjunction with conventional wet chemical methods. Plasma surface modification is particularly interesting because the method is flexible, rapid, contaminant-free, and relatively nondestructive $[10,11]$. The excited species (ions, radicals, and electrons) and UV light within the plasma interact with the surfaces of CNTs and break the $\mathrm{sp}^{2}$-hybridized carbon $(\mathrm{C}=\mathrm{C})$ bonds creating the prime reaction sites [10-12]. A wide range of functional groups can then be introduced onto the surfaces of CNTs depending on the plasma production method, the source gas such as $\mathrm{O}_{2}, \mathrm{~N}_{2}, \mathrm{NH}_{3}$, or $\mathrm{H}_{2} \mathrm{O}$, the input power, and the processing time [10-13].

In this study, we have attempted to modify multiwalled carbon nanotubes (MWCNTs) by three-step processing. MWCNTs are first supersonically mixed into ethanol to be deaggregated temporarily. Then, the mixture is dried and pretreated using citric acid solution. Finally, the MWCNTs in the solution are treated by the oxygen plasma including citric acid and water. This method is safer than the methods mentioned above as no hazardous reagents are used. The surfaces of the MWCNTs are chemically functionalized with carboxyl $(-\mathrm{COOH})$ groups, and they can be easily dispersed 


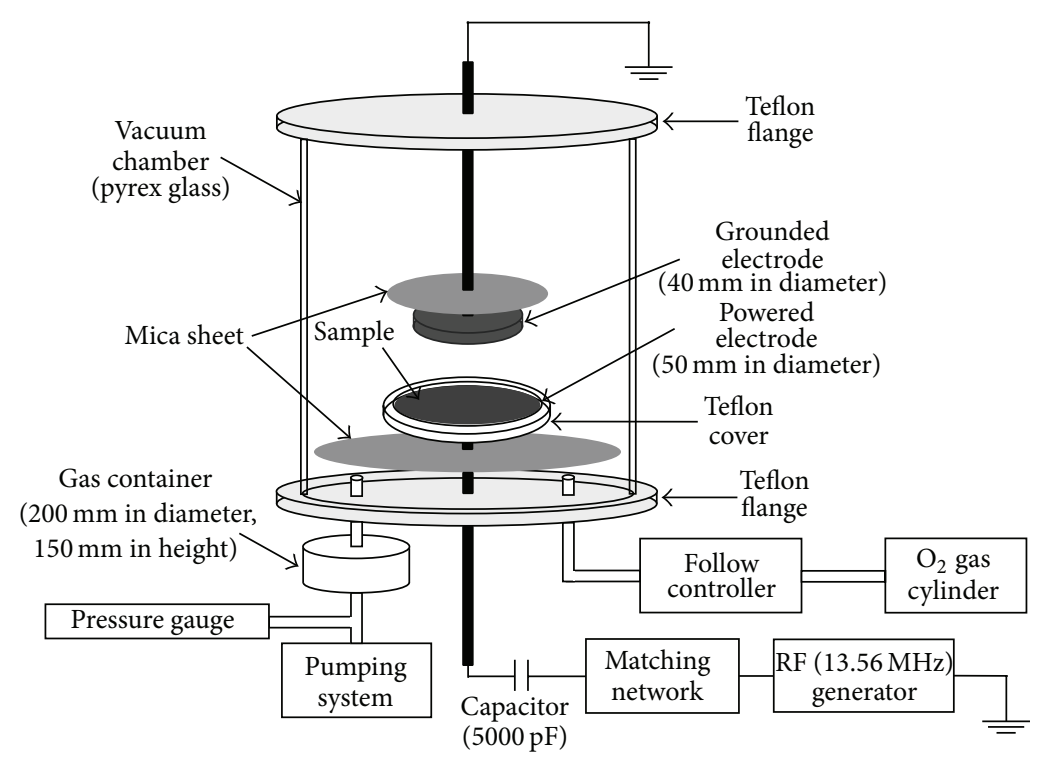

(a)

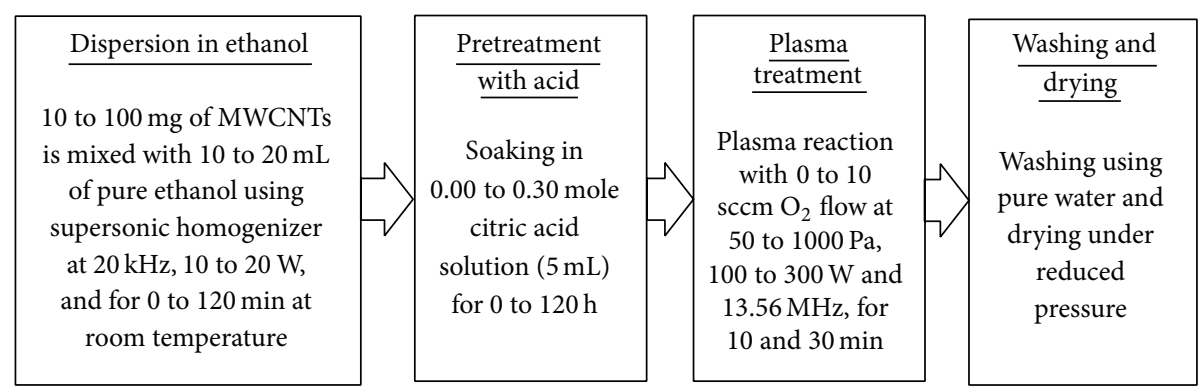

(b)

FIGURE 1: (a) Schematic diagram of the plasma reactor. (b) Flow chart of the functionalization process.

in water. Each step of the functionalization processes is optimized by varying the treatment parameters.

\section{Experimental Setup and Method}

A schematic setup of the plasma reactor and a flow chart of the functionalization process are shown in Figure 1.10 to $100 \mathrm{mg}$ of MWCNT powder (Sigma-Aldrich, outer diameter $=10$ to $30 \mathrm{~nm}$; inner diameter $=3$ to $10 \mathrm{~nm}$; length $=1$ to $10 \mu \mathrm{m}$; purity $>90 \%$ ) is added to 10 to $20 \mathrm{~mL}$ of pure ethanol (Wako Pure Chemicals Co., purity $>95 \%$ ) and sonicated at room temperature using a supersonic homogenizer (Sonics Vibra cell, VC 130, Sonic \& Materials Inc., $f=20 \mathrm{kHz}, 6.0 \mathrm{~mm} \phi$ probe) at an input power of 10 to $20 \mathrm{~W}$ for 15 to $120 \mathrm{~min}$. The suspension is dried under reduced pressure and soaked in 0.0 to 0.30 mole $(5 \mathrm{~mL})$ of citric acid (Wako Pure Chemicals Co., assay $>98 \%$ ) solution for 0 to $120 \mathrm{~h}$. The MWCNTs in the citric acid solution are then placed on the lower electrode (SUS, $50 \mathrm{~mm} \phi$ ) of the reactor, which is evacuated to ca. $400 \mathrm{~Pa}$ using a rotary pump at a very slow rate. When the wet phase starts to disappear, oxygen gas is introduced into the reactor at a rate of 0 to $10 \mathrm{sccm}$ and the background chamber pressure is kept at about $400 \mathrm{~Pa}$. Then the plasma reaction is carried out for 10 to $30 \mathrm{~min}$ by an RF input power of $P_{\text {rf }}=100-300 \mathrm{~W}, f=13.56 \mathrm{MHz}$. The reflected RF power is minimized $(<20 \mathrm{~W})$ by controlling the matching current during the plasma reaction. It is noted that when the samples become fully dried before starting the plasma reaction, they are less reactive for the oxygen plasma. However, when the plasma is started in the wet phase the water molecules and part of the citric acid molecules evaporate with the processing time but are considered to remain inside the chamber and in the gas container connected to the chamber, contributing to the functionalization process. After the treatment, the MWCNTs are washed at least three times using pure water (Wako Pure Chemicals Co., distilled water) and dried under reduced pressure at room temperature.

Fourier transform infrared (FT-IR) spectroscopy is used to measure the chemical groups attached onto the MWCNTs. Approximately $0.5 \mathrm{mg}$ of a dried sample is dispersed in $1.0 \mathrm{~mL}$ of propanol, and the mixture is uniformly coated on a $\mathrm{CaF}_{2}$ substrate (SIGMA KOKI Co., $20 \mathrm{~mm}$ diameter and $1 \mathrm{~mm}$ thickness), dried, and measured using an FT-IR spectrometer (Shimadzu Co., 8700, 100 scans averaged). The spectra presented in this report are obtained after base line correction.

To study the dispersion stability of the pristine, sonicated, and plasma-treated MWCNTs (hereinafter denoted 


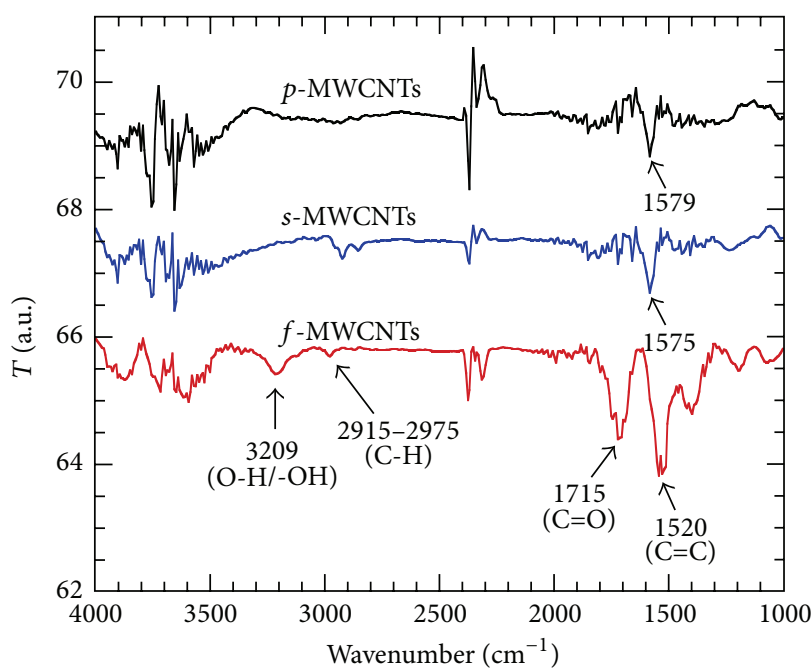

(a)

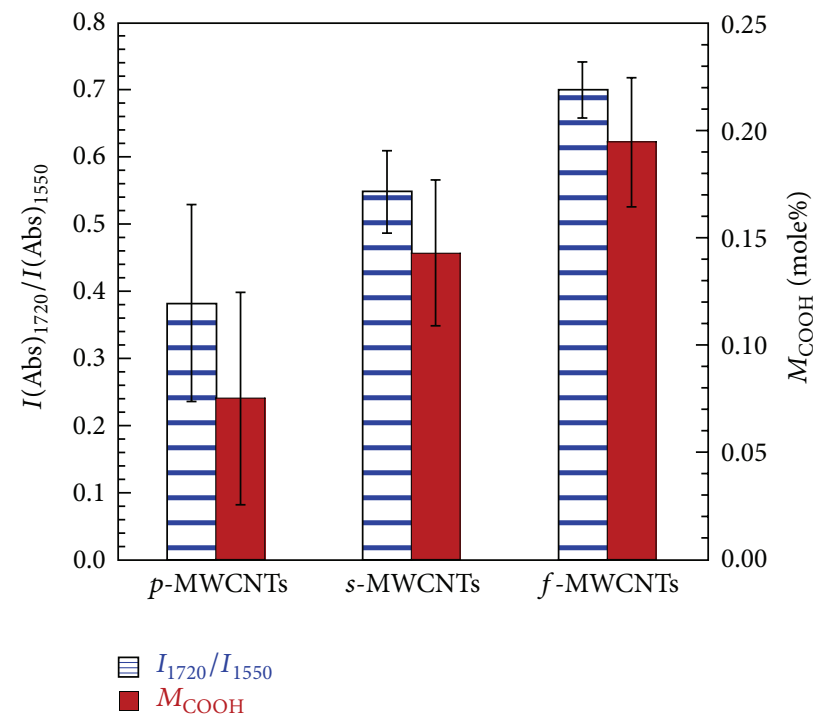

(b)

FIGURE 2: (a) FT-IR spectra for the $p$-MWCNTs, $s$-MWCNTs, and $f$-MWCNTs and (b) intensity ratios of the FT-IR absorbance peak at ca. 1720 to the most intense peak in the range $1520-1590 \mathrm{~cm}^{-1}$ and the content of carboxyl groups, $M_{\mathrm{COOH}}$ (mole\%), estimated from the absorbance peak intensities at ca. $1720 \mathrm{~cm}^{-1}$ using the calibration curve drawn for benzoic acid.

by $p$-MWCNTs, $s$-MWCNTs, and $f$-MWCNTs, resp.), ca. $1.0 \mathrm{mg}$ of the dried sample is mixed with $8.0 \mathrm{~mL}$ of pure water with a brief sonication for $2 \mathrm{~min}$ in a bath sonicator (IUCHI Japan, US-1, $110 \mathrm{~W}, 38 \mathrm{kHz}$ ) so that the MWCNTs are dispersed homogeneously. The dispersion stability is examined by a UV-visible spectrometer (JASCO Co., V-630). The sample is placed in a quartz cuvette $\left(1.0 \times 1.0 \times 4.5 \mathrm{~cm}^{3}\right)$ and time variation of absorbance (Abs) at a wavelength of $250 \mathrm{~nm}$ is measured for $4 \mathrm{~h}$. Here the wavelength is chosen corresponding to the maximum absorbance region of the UV-visible spectra, because Beer's law of the linear relationship between the absorbance and concentration of the absorbing particles holds up to higher concentration in this region as compared to other regions [14]. The dispersion stability is also verified by mixing $20 \mathrm{mg}$ of each sample in $10 \mathrm{~mL}$ of pure water with bath sonication for $2 \mathrm{~min}$ and then keeping the sample in a glass bottle undisturbed for more than one month.

The surface morphology of the samples is observed by a transmission electron microscope (TEM; HITACHI High Technology Co., H-7500), and the structural quality is measured by a Raman spectrometer (JASCO Co., NR-1800, $\lambda=532 \mathrm{~nm})$.

\section{Results and Discussion}

A variety of different techniques can be used to characterize the surface chemistry of CNTs after covalent functionalization $[9,12,15,16]$. Among them FT-IR is widely used to ascertain the groups attached onto the functionalized CNTs $[16,17]$. Therefore, FT-IR spectra of the $p$-MWCNTs, $s$-MWCNTs, and $f$-MWCNTs are measured. Usually, $p$-MWCNTs exhibit an almost featureless FT-IR spectrum because of the high transmittance of the $\pi$-electron structure of symmetric carbons [18]. But in our case a small peak at approximately $1579 \mathrm{~cm}^{-1}$ is observed, which corresponds to $\mathrm{C}=\mathrm{C}$ stretching vibration as shown in Figure 2(a) $[16,19] . s$-MWCNTs do not show significant difference in the spectrum from that of the p-MWCNTs except for C$\mathrm{H}$ stretching peaks at approximately 2925 to $2975 \mathrm{~cm}^{-1}$, indicating an increase in the number of $\mathrm{sp}^{3}$ carbon bonds $[16,17,20]$. These bonds are assumed to originate from the breakage of $\mathrm{C}=\mathrm{C}$ bonds. A new clear peak appears at ca. $1720 \mathrm{~cm}^{-1}$ in the spectrum of $f$-MWCNTs as shown in Figure 2(a), which indicates the presence of $\mathrm{C}=\mathrm{O}$ stretching bonds, and the broad band at ca. $3209 \mathrm{~cm}^{-1}$ corresponds to $\mathrm{O}-\mathrm{H} /-\mathrm{OH}$ bonds $[18,19]$. Hence, it is conjectured that after the plasma treatment MWCNTs are functionalized and that hydrophilic carboxyl $(-\mathrm{COOH})$ groups are attached onto the MWCNTs. Usually $\mathrm{C}=\mathrm{O}$ stretching vibrations of the $\mathrm{COOH}$ group are observed at $1740 \mathrm{~cm}^{-1}$ [8], but in our case the $\mathrm{C}=\mathrm{O}$ vibration peak is shifted to ca. $1720 \mathrm{~cm}^{-1}$, which suggests the abundance of $-\mathrm{COOH}$ groups on the MWCNTs after plasma treatment [21]. The peak at 1520 to $1590 \mathrm{~cm}^{-1}$ corresponds to the $\mathrm{C}=\mathrm{C}$ graphitic stretching mode, which is infrared-activated by extensive sidewall functionalization $[16,21]$. When $f$-MWCNTs are dispersed in water, the $-\mathrm{COOH}$ groups attached to the MWCNT surface are ionized to $\mathrm{COO}^{-}$. As a result, the negative charges repel each other and the MWCNTs do not aggregate in the solvent. Thus, a stable dispersed solution is produced from the $f$-MWCNTs.

For the quantitative study of the attached groups different methods such as X-ray photoelectron spectroscopy (XPS), thermogravimetric analysis (TGA), chemical derivatization, or titration methods are used $[9,13,15]$. Each of these methods has strengths as well as limitations in finding the exact amount of the groups [22]. In this study, absorbance 


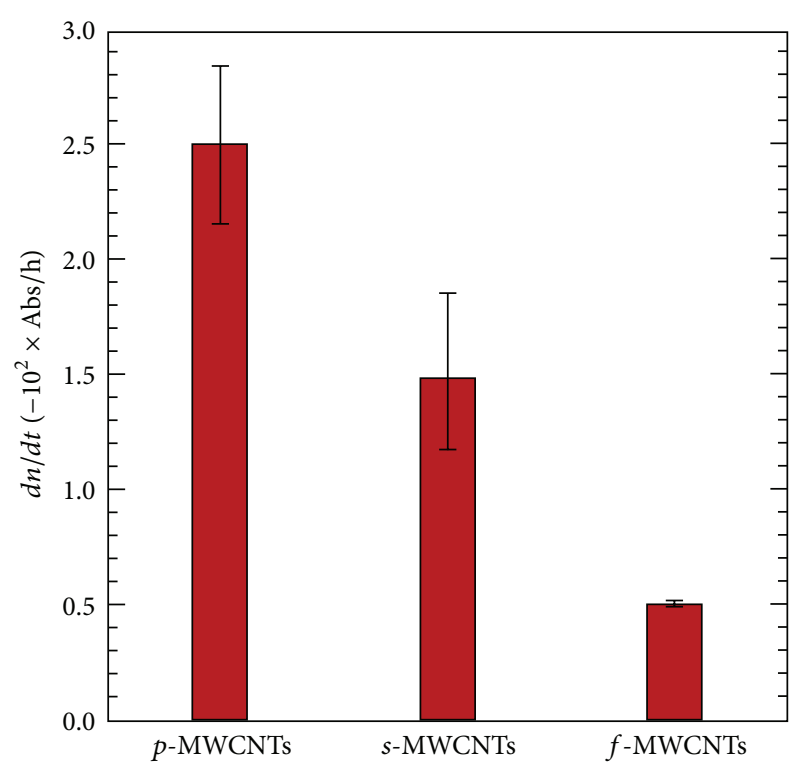

(a)

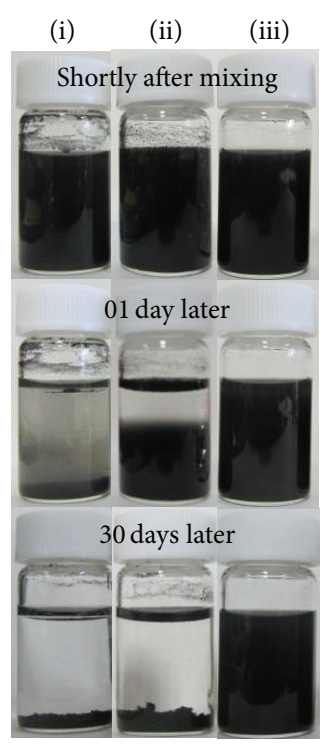

(b)

FIGURE 3: (a) Settling speed, $d n / d t$, calculated from the $A b s(250 \mathrm{~nm})$ versus time graphs for the $p$-MWCNTs, $s$-MWCNTs, and $f$-MWCNTs. (b) Photographs of the dispersion of $20 \mathrm{mg}$ of (i) $p$-MWCNTs, (ii) $s$-MWCNTs, and (iii) $f$-MWCNTs in $10 \mathrm{~mL}$ of pure water using 2 min of bath sonication.

intensity of the $\mathrm{C}=\mathrm{O}$ stretching peak in the FT-IR spectra is considered to roughly estimate the quantity of $-\mathrm{COOH}$ groups attached onto the MWCNT surfaces. For this, a calibration curve is drawn from the absorbance spectra of a set of benzoic acid $\left(\mathrm{C}_{6} \mathrm{H}_{5}-\mathrm{COOH}\right)$ solutions of different known concentrations. As $\mathrm{C}_{6} \mathrm{H}_{5}-\mathrm{COOH}$ structure has only one $-\mathrm{COOH}$ group in the benzene ring, the intensity of the $\mathrm{C}=\mathrm{O}$ stretching bond is considered to be a measure of $-\mathrm{COOH}$ content in the solution. The equation of the calibration curve is

$$
y=2.0963 x
$$

where $x$ is the molar concentration and $y$ is the corresponding intensity of the absorbance peak at ca. $1720 \mathrm{~cm}^{-1}$, indicating the $\mathrm{C}=\mathrm{O}$ content of $-\mathrm{COOH}$ groups [19]. Then, using the calibration curve, molar percentages of the $-\mathrm{COOH}$ $\left(M_{\mathrm{COOH}}\right)$ attached onto the MWCNT surfaces are roughly estimated from the peak intensities of the absorbance data of the $p$-MWCNTs, $s$-MWCNTs, and $f$-MWCNTs at ca. $1720 \mathrm{~cm}^{-1}$ and are shown in Figure 2(b). The ratios of the peak intensity at ca. $1720 \mathrm{~cm}^{-1}$ and that of the intense peak in the range 1520 to $1590 \mathrm{~cm}^{-1}, I_{1720} / I_{1550}$, are also calculated to confirm the content of $-\mathrm{COOH}$ groups. It is observed that $p$-MWCNTs contain a small amount of $-\mathrm{COOH}$, which may originate from their purification process. The content is slightly higher for $s$-MWCNTs, which may be due to the interaction of ethanol with MWCNTs under the cavitation forces in the sonication process. The significant increase in $\mathrm{C}=\mathrm{O}$ content and the increase in $I_{1720} / I_{1550}$ after the plasma treatment indicate the functionalization of MWCNTs. It is conjectured that during the acidic pretreatment, citric acid molecules become wrapped around the MWCNTs. From the Raman spectra (described later), it is observed that the $p$-MWCNTs have defect sites on their structure. Therefore, there is a chance for the citrate ions to slowly attack the weak parts of the MWCNTs. As a result, some primary reaction sites may temporarily become functionalized. Besides, a large number of active sites are assumed to be generated on the surfaces of the MWCNTs from the attack of energetic ions and radicals produced during the plasma treatment. The oxygen ions and radicals are highly reactive and can form covalent bonds upon reacting with dangling bonds or defects [12]. The active sites then become attached to $-\mathrm{OH}$ or $\mathrm{COOH}$ groups, which are generated from the fragmentation of the citric acid, water, and oxygen.

The surface functional groups increase the dispersion stability of MWCNTs. Hence, dispersion stability can be an indirect measure of the degree of MWCNT functionalization. UV-visible spectroscopy is used to study the dispersion stability of the treated samples in pure water. The absorption of UV-visible light is quantitatively highly accurate [14] and the change in absorbance with time can be interpreted as dispersion stability of MWCNTs in water. Because the absorbance of the solution is proportional to the concentration of the absorbing particles in the solvent [14], the settling speed $(d n / d t)$, measured from the slope of the linear fitting of the Abs versus time graph, would give the information about the amount of MWCNTs present in the optical path. Settling speed was observed to be used as a measure of studying the functionalized MWCNTs [23]. A decrease in $d n / d t$ represents an increase in the dispersion stability of MWCNTs in the solvent.

The settling speeds (with error bars) of the p-MWCNTs, $s$-MWCNTs, and $f$-MWCNTs in water are shown in Figure 3(a), which are averaged from at least three times 


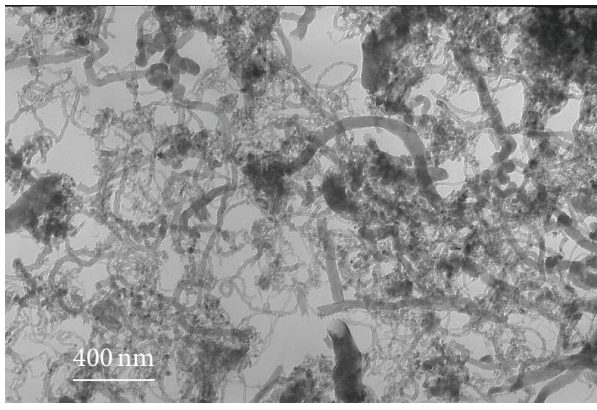

(a)

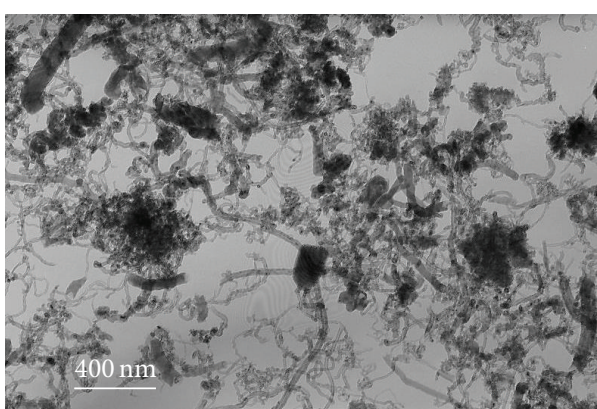

(c)

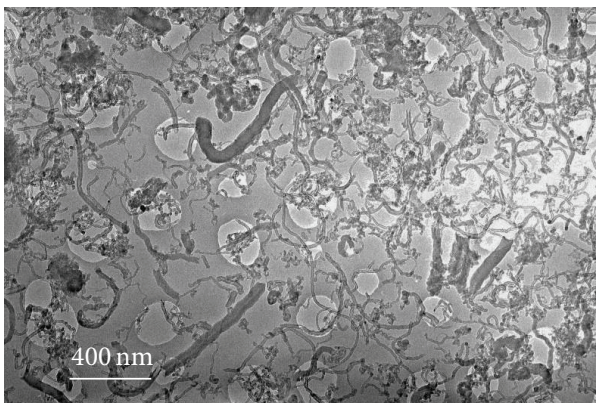

(e)

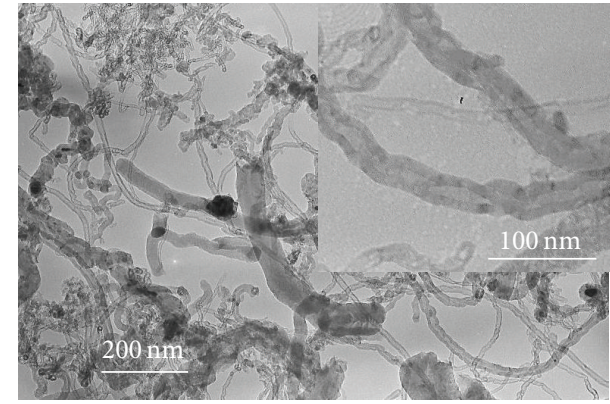

(b)

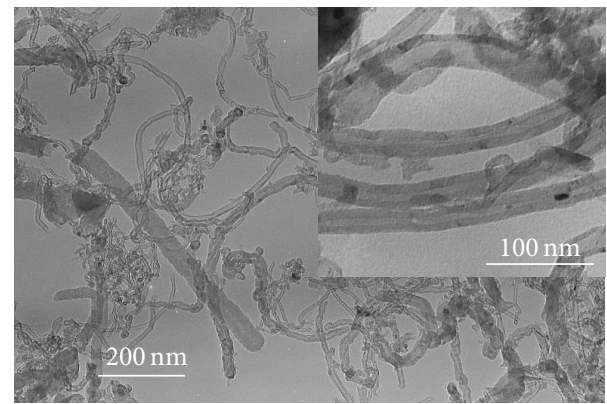

(d)

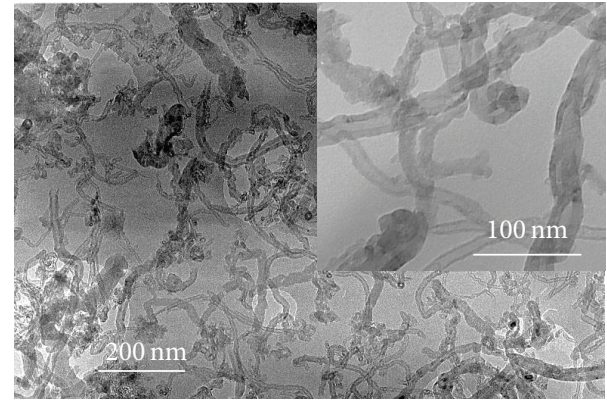

(f)

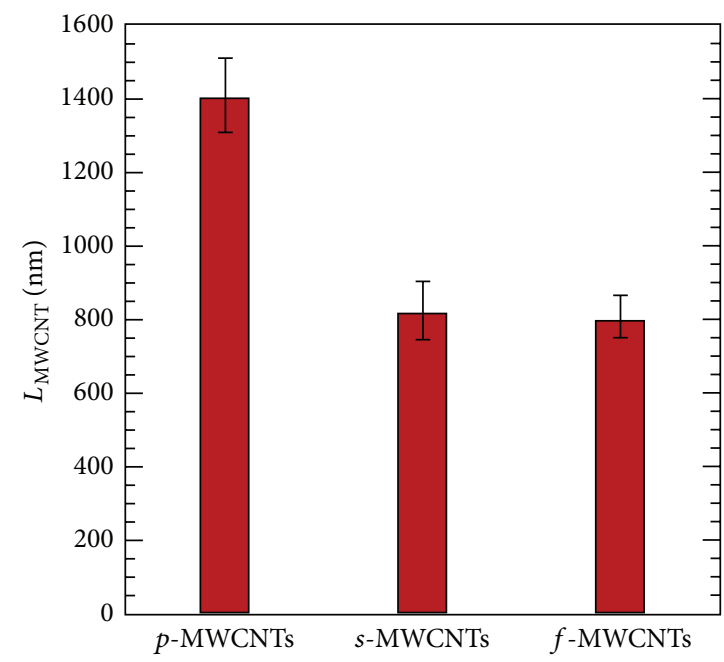

(g)

Figure 4: TEM images of the (a) and (b) $p$-MWCNTs, (c) and (d) $s$-MWCNTs, and (e) and (f) $f$-MWCNTs at different magnifications taken at an accelerating voltage of $100 \mathrm{kV}$. Insets of (b), (d), and (f) are showing higher magnification images. (g) Average MWCNT length $\left(L_{\mathrm{MWCNT}}\right)$ measured from more than 30 MWCNTs in each sample from several TEM images. 
of the measurement. The lower value of $d n / d t$ for the $s$-MWCNTs than for the $p$-MWCNTs indicates that the dispersibility is enhanced slightly by sonication. However, after the citric-acid-assisted plasma treatment, $d n / d t$ becomes one-fifth that of the $p$-MWCNTs, which indicates the strong enhancement of dispersibility. The dispersion stability can also be observed in the optical photographs shown in Figure $3(\mathrm{~b})$. It is observed that the $f$-MWCNTs produce a darker suspension than $p$-MWCNTs or $s$-MWCNTs, and the little change in the darkness even after a week indicates the higher dispersibility of $f$-MWCNTs. $p$-MWCNTs agglomerate soon after they are dispersed in water and form loose sediment on the container bottom. A part of the MWCNTs is assumed to be broken into shorter pieces during the supersonic treatment, and they are less likely to become entangled and aggregate [24]. As a result, the dispersibility of $s$-MWCNTs is improved. On the other hand, the $f$-MWCNTs disperse easily in water and the suspension remains stable for more than one month confirming the enhanced dispersibility.

The morphology of the MWCNTs is observed by TEM at each step of functionalization as shown in Figures 4(a)-4(e). The typical TEM images indicate that $f$-MWCNTs are more dispersed than $p$-MWCNTs and $s$-MWCNTs. Shortening of the MWCNT length is observed as a result of sonication, but almost no further shortening is observed after the subsequent plasma treatment. The plasma species only affect the surfaces of the MWCNTs and not the bulk [12]. No significant defects are observed on the sidewalls of $f$-MWCNTs in the TEM images of higher magnification, which suggest the preservation of structural integrity.

Raman spectrum is an important tool to study the CNT structure $[25,26]$ and is widely used to assess the amount of defects $[16,17]$. Raman spectra of the $p$-MWCNTs, $s$-MWCNTs, and $f$-MWCNTs are presented in Figure 5, which are normalized and bodily shifted in the intensity axis. The defect induced $D$ band appears at ca. $1350 \mathrm{~cm}^{-1}$, which indicates the amount of disordered carbon in the CNT structure, and its intensity, $I_{D}$, corresponds to the degree of disorderness. The $G$ band at ca. $1600 \mathrm{~cm}^{-1}$ corresponds to the graphitic (ordered) carbon, and its intensity, $I_{G}$, corresponds to the amount of ordered carbon. Therefore, the ratio, $I_{D} / I_{G}$, is used to estimate the change in structural quality of CNTs after functionalization in different processes. Defect density corresponding to $I_{D} / I_{G}$ for the $p$-MWCNTs, $s$-MWCNTs, and $f$-MWCNTs is observed to be $1.05 \pm 0.02,1.06 \pm 0.01$, and $1.08 \pm 0.03$, respectively. The changes are very small, and also no distinct changes are observed in terms of the Raman shift. These suggest that the MWCNT structure and the chemical composition of the interior of the CNTs are almost unaffected by the ultrasonic and plasma treatments. This is also supported by the results of the TEM measurement.

On the basis of the above results, the basic functionalization scheme of the MWCNTs by the citric-acid-assisted oxygen plasma treatment is summarized in Figure 6. MWCNTs are long and web-like and remain strongly aggregated. When they are dispersed in ethanol by the supersonic treatment, ethanol molecules enter the aggregated parts of the MWCNTs and weaken the attractive forces between them. When the

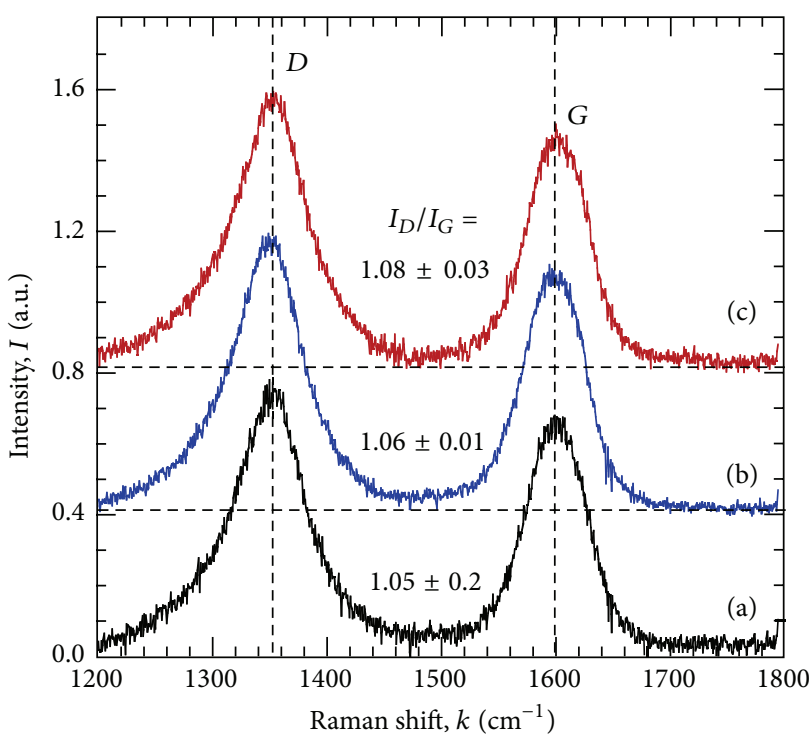

Figure 5: Raman spectra of the (a) p-MWCNTs, (b) $s$-MWCNTs, and (c) $f$-MWCNTs.

$s$-MWCNTs are placed in the citric acid solution, the citrate and hydronium ions attack their weak parts. During the plasma reaction, oxygen, water, and citric acid molecules or ions are fragmented to generate oxygen containing ions, radicals, and $\mathrm{CO}$ or $\mathrm{CO}_{2}$, which react with the defect sites $[12,27,28]$. The $\mathrm{CO}$ and $\mathrm{CO}_{2}$ are oxidized to form $-\mathrm{COOH}$ groups and attach to the MWCNT surfaces [18]. Also, the attached $-\mathrm{OH}$ groups are further oxidized to form $-\mathrm{COOH}$ groups $[17,28]$. These functional groups enable the MWCNTs to readily disperse in water due to hydrogen bonds formed between the carboxylic acid groups and water molecules [29]. The negatively charged surfaces of the CNTs repel each other and prevent them from coagulating. The polar interactions of the functional groups with the water molecules reduce the settling speed of the $f$-MWCNTs [29].

3.1. Optimization of the Functionalization Process. The dispersion stability depends on the degree of functionalization. Therefore, the settling speed shows qualitatively the content of functional groups attached onto the MWCNT surfaces. Using this, optimal treatment conditions are sought for each step of the processes.

Sonication parameters play very important role in the dispersion of CNTs [30, 31]. To optimize the input sonication power, 10,15 , and $20 \mathrm{~W}$ are considered for the treatment of $30 \mathrm{mg}$ of MWCNTs in $20 \mathrm{~mL}$ of pure ethanol for $60 \mathrm{~min}$. For optimum treatment time, $30 \mathrm{mg}$ of MWCNTs in $20 \mathrm{~mL}$ of ethanol is treated for $15,30,60,90$, and $120 \mathrm{~min}$ at an input power of $15 \mathrm{~W}$. MWCNTs of $10,30,50$, and $100 \mathrm{mg}$ in $20 \mathrm{~mL}$ of pure ethanol are treated at $15 \mathrm{~W}$ for $60 \mathrm{~min}$ to optimize the amount in ethanol. Temperature of the mixture is kept constant by placing the flask containing the sample in a bath of ice water during the sonication to facilitate the equilibrium condition for the treatment [31]. The $d n / d t$ is measured for all of these samples as described earlier and presented in 


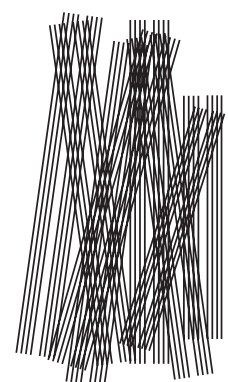

Pristine MWCNTs (highly coagulated)

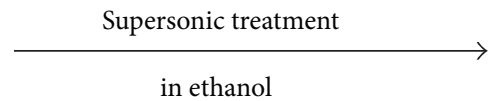

in ethanol

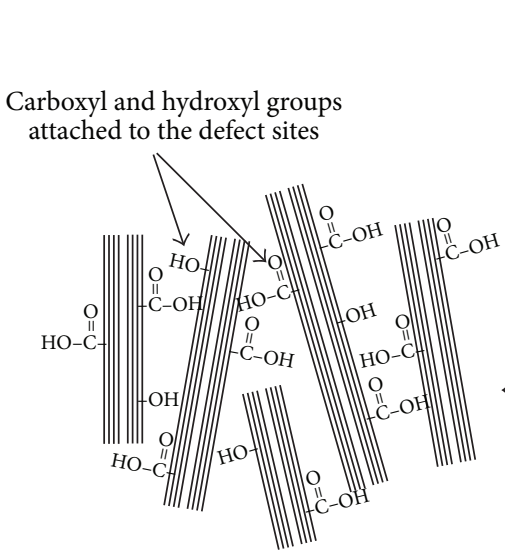

Plasma-treated MWCNTs (functionalized by $-\mathrm{COOH}$ and $-\mathrm{OH}$ groups)

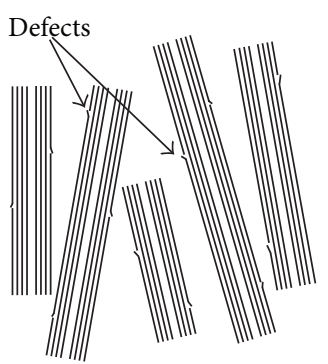

Sonicated MWCNTs (shortened and decoagulated)

Pretreatment by citric acid

FIGURE 6: A model of the functionalization process of MWCNTs.

Figure 7(a), based on which it is observed that treatment of $30 \mathrm{mg}$ of MWCNTs in $20 \mathrm{~mL}$ of ethanol at an input power of $15 \mathrm{~W}$ for $60 \mathrm{~min}$ is optimum for supersonic treatment.

The degree of plasma functionalization changes with the citric acid density and the soaking time. To optimize these, $s$-MWCNTs sonicated at optimum condition are placed in $5 \mathrm{~mL}$ of citric acid solution of $0.10,0.15,0.20$, and 0.30 mole for $48 \mathrm{~h}$. For optimum soaking time $s$-MWCNTs are soaked in 0.15 mole $(5 \mathrm{~mL})$ citric acid solutions for $0,24,48$, and $120 \mathrm{~h}$. After soaking, each sample is plasma treated for $15 \mathrm{~min}$ at $200 \mathrm{~W}$ with $\mathrm{O}_{2}$ flow of $5 \mathrm{sccm}$. Then $d n / d t$ is measured for these $f$-MWCNTs, which are presented in Figure 7(b). It is observed that soaking in 0.15 mole citric acid solution for ca. $48 \mathrm{~h}$ is optimum for citric acid treatment. Though $d n / d t$ decreases for the samples soaked for longer time, 24 to $48 \mathrm{~h}$ is considered as sufficient, because after this the changes in the $d n / d t$ are not so high.

To optimize the plasma reaction condition three different $\mathrm{O}_{2}$ flow rates are considered $(0,5$, and $10 \mathrm{sccm})$, at which the samples are plasma treated at a pressure of ca. $400 \mathrm{~Pa}$ and $P_{\mathrm{rf}}$ of $200 \mathrm{~W}$ for $15 \mathrm{~min}$. Similarly, background pressure is varied from 50 to $1000 \mathrm{~Pa}$ for the treatment at $P_{\mathrm{rf}}=$ $200 \mathrm{~W}$ for $15 \mathrm{~min}$ with $5 \mathrm{sccm} \mathrm{O}_{2}$ flow. RF power is varied as 100,200 , and $300 \mathrm{~W}$ at a pressure of $400 \mathrm{~Pa}$ with $\mathrm{O}_{2}$ flow of $5 \mathrm{sccm}$ and treatment time of $15 \mathrm{~min}$. The treatment duration is varied as $10,15,20$, and 30 min with $\mathrm{O}_{2}$ flow of $5 \mathrm{sccm}$ and $P_{\mathrm{rf}}$ of $200 \mathrm{~W}$ with background pressure of $400 \mathrm{~Pa}$. From the values of $d n / d t$ for all these samples, which is shown in Figure 7(c), it is observed that $5 \mathrm{sccm}$ $\mathrm{O}_{2}$ flow, $400 \mathrm{~Pa}$ background pressure, $200 \mathrm{~W}$ RF power, and ca. 15 min treatment duration are optimum for the efficient functionalization. Plasma treatment without an $\mathrm{O}_{2}$ flow is found to be less efficient. On the other hand, a higher $\mathrm{O}_{2}$ flow rate $(10 \mathrm{sccm})$ is also observed to deteriorate the functionalization. Higher RF power $(300 \mathrm{~W})$ is also observed to deteriorate the dispersion stability, which is assumed to be due to the dissociation of the functional groups to other groups. In the same way, $15 \mathrm{~min}$ of plasma treatment at $200 \mathrm{~W}$ has a more positive effect on functionalization than $10 \mathrm{~min}$ treatment and 20 or 30 min treatment results in no significant enhancement of functionalization. It is conjectured that after 15 min of treatment the plasma reaction reaches a saturation level, and therefore further treatment cannot improve the functionalization.

For clear results on the quantitative measurement of the attached groups XPS analysis is necessary, which will be done in the near future. As the plasma comes in from the upper side, surface part of the samples on the electrode may react more strongly than the inner part. Therefore, to obtain uniform functionalization, modification of the reactor configuration would be necessary, which is under active consideration. 


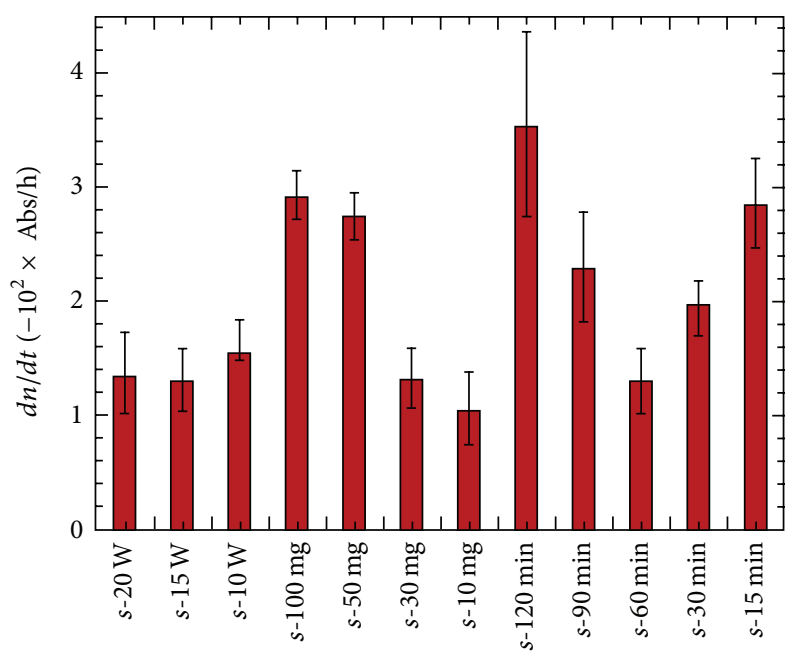

(a)

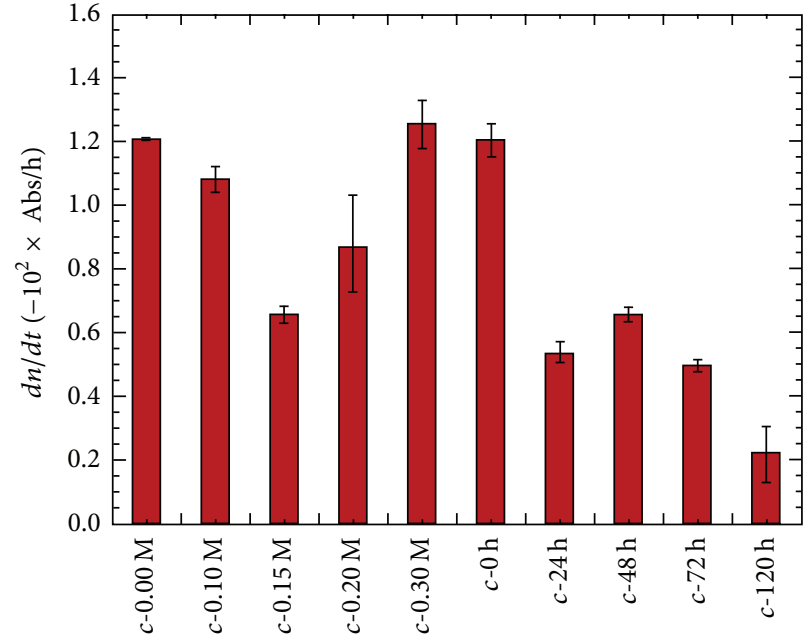

(b)

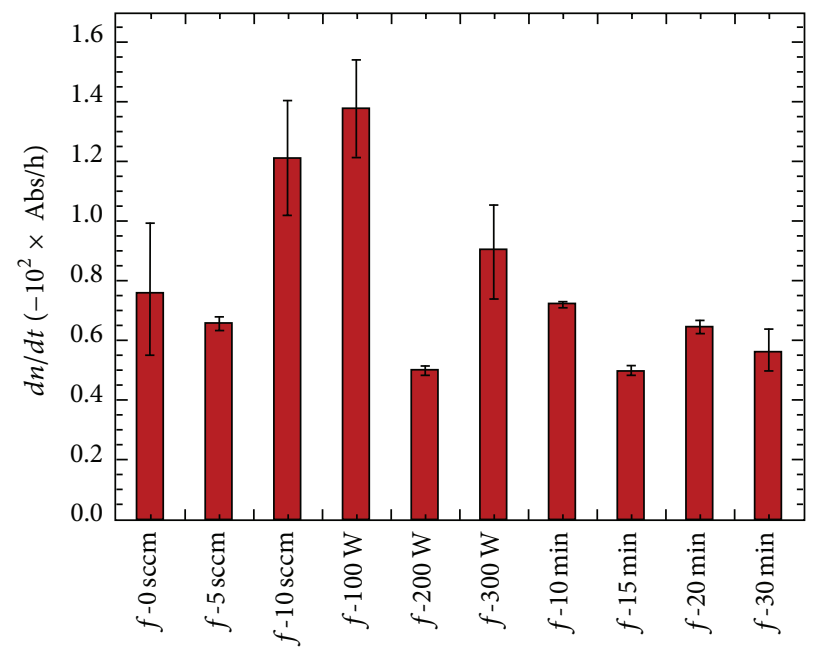

(c)

Figure 7: Settling speeds, $d n / d t$, measured (a) for the $s$-MWCNTs treated supersonically at different powers (10 to $15 \mathrm{~W}$ ), different amounts (10 to $100 \mathrm{mg}$ ) in $20 \mathrm{~mL}$ ethanol, and different durations (15 to $120 \mathrm{~min}$ ) at room temperature, (b) for the plasma treated samples soaked in different concentrations of citric acid (0.00-3.0 M), and for different durations $(0$ to $120 \mathrm{~h})$ at room temperature, and (c) for the $f$-MWNTs plasma treated at different oxygen flows (0 to $10 \mathrm{sccm}$ ), RF power (100 to $300 \mathrm{~W}$ ), and for different durations (10 to $30 \mathrm{~min}$ ).

\section{Conclusions}

A new and safe method has been developed to functionalize MWCNTs. In the sequence of treatments, MWCNTs are pretreated in pure ethanol using a supersonic homogenizer, wetted using citric acid solution, and plasma treated using RF oxygen plasma. By the plasma reaction in the presence of citric acid, $\mathrm{O}_{2}$, and water vapor, plasma species interact with them to create many kinds of ions and radicals. They attack the MWCNT surfaces and activate a large number of sites to enhance the attachment of $-\mathrm{COOH}$ groups onto their surfaces. These attached groups significantly enhance the dispersion stability of the MWCNTs in water. The structural integrity of the $f$-MWCNTs is conjectured to be preserved after plasma treatment, which is verified by the Raman and TEM measurements. The most suitable treatment parameters in this process are the sonication of $30 \mathrm{mg}$ MWCNTs in $20 \mathrm{~mL}$ ethanol at room temperature for $60 \mathrm{~min}$ at a probe power of $15 \mathrm{~W}$, soaking in 0.15 mole $(5 \mathrm{~mL})$ citric acid solution for ca. $48 \mathrm{~h}$, and plasma treatment for ca. $15 \mathrm{~min}$ at a pressure of ca. $400 \mathrm{~Pa}$ and an RF power of $200 \mathrm{~W}$ with ca. $5 \mathrm{sccm} \mathrm{O}$ flow.

\section{Conflict of Interests}

The authors declare that there is no conflict of interests regarding the publication of this paper.

\section{Acknowledgments}

This study is partly supported by the Promotion of NanoBiotechnology Research to Support Aging, Welfare Society from Ministry of Education, Culture, Sports, Science and Technology, Japan. The authors would like to thank 
Mr. Waichi Tomoda and Ms. Yumiko Sugaya for their technical support.

\section{References}

[1] R. H. Baughman, A. A. Zakhidov, and W. A. De Heer, "Carbon nanotubes-the route toward applications," Science, vol. 297, no. 5582, pp. 787-792, 2002.

[2] M. Endo, M. S. Strano, and P. M. Ajayan, "Potential applications of carbon nanotubes," Carbon Nanotubes, vol. 111, pp. 13-62, 2008.

[3] D. Tasis, N. Tagmatarchis, A. Bianco, and M. Prato, "Chemistry of carbon nanotubes," Chemical Reviews, vol. 106, no. 3, pp. 1105-1136, 2006.

[4] A. Hirsch and O. Vostrowsky, "Functionalization of carbon nanotubes," Topics in Current Chemistry, vol. 245, pp. 193-237, 2005.

[5] J. Liu, A. G. Rinzler, H. Dai et al., "Fullerene pipes," Science, vol. 280, no. 5367, pp. 1253-1256, 1998.

[6] M. Pavese, S. Musso, S. Bianco, M. Giorcelli, and N. Pugno, "An analysis of carbon nanotube structure wettability before and after oxidation treatment," Journal of Physics Condensed Matter, vol. 20, no. 47, Article ID 474206, 2008.

[7] K. J. Ziegler, Z. Gu, H. Peng, E. L. Flor, R. H. Hauge, and R. E. Smalley, "Controlled oxidative cutting of single-walled carbon nanotubes," Journal of the American Chemical Society, vol. 127, no. 5, pp. 1541-1547, 2005.

[8] K. A. Worsley, I. Kalinina, E. Bekyarova, and R. C. Haddon, "Functionalization and dissolution of nitric acid treated singlewalled carbon nanotubes," Journal of the American Chemical Society, vol. 131, no. 50, pp. 18153-18158, 2009.

[9] K. Peng, L. Liu, H. Li, H. Meyer, and Z. Zhang, "Room temperature functionalization of carbon nanotubes using an ozone/water vapor mixture," Carbon, vol. 49, no. 1, pp. 70-76, 2011.

[10] A. Felten, C. Bittencourt, J. J. Pireaux, G. Van Lier, and J. C. Charlier, "Radio-frequency plasma functionalization of carbon nanotubes surface $\mathrm{O}_{2}, \mathrm{NH}_{3}$, and $\mathrm{CF}_{4}$ treatments," Journal of Applied Physics, vol. 98, no. 7, Article ID 074308, 2005.

[11] C. H. Chiu, C. C. Lin, H. V. Han et al., "High efficiency GaN-based light-emitting diodes with embedded air voids/ $\mathrm{SiO}_{2}$ nanomasks," Nanotechnology, vol. 23, no. 4, Article ID 045303, 2012.

[12] C. Chen, B. Liang, D. Lu, A. Ogino, X. Wang, and M. Nagatsu, "Amino group introduction onto multiwall carbon nanotubes by $\mathrm{NH}_{3} /$ Ar plasma treatment," Carbon, vol. 48, no. 4, pp. 939948, 2010.

[13] C. Chen, B. Liang, A. Ogino, X. Wang, and M. Nagatsu, "Oxygen functionalization of multiwall carbon nanotubes by microwaveexcited surface-wave plasma treatment," Journal of Physical Chemistry C, vol. 113, no. 18, pp. 7659-7665, 2009.

[14] T. Owen, Fundamentals of UV-Visible Spectroscopy, HewlettPackard, Böblingen, Germany, 1996.

[15] S. Visentin, N. Barbero, S. Musso et al., "A sensitive and practical fluorimetric test for CNT acidic site determination," Chemical Communications, vol. 46, no. 9, pp. 1443-1445, 2010.

[16] R. Tian, X. Wang, M. Li et al., "An efficient route to functionalize singe-walled carbon nanotubes using alcohols," Applied Surface Science, vol. 255, no. 5, pp. 3294-3299, 2008.

[17] D. G. Tong, Y. Y. Luo, W. Chu, Y. C. Guo, and W. Tian, "Cutting of carbon nanotubes via solution plasma processing," Plasma
Chemistry and Plasma Processing, vol. 30, no. 6, pp. 897-905, 2010.

[18] M. V. Naseh, A. A. Khodadadi, Y. Mortazavi, F. Pourfayaz, O. Alizadeh, and M. Maghrebi, "Fast and clean functionalization of carbon nanotubes by dielectric barrier discharge plasma in air compared to acid treatment," Carbon, vol. 48, no. 5, pp. 13691379, 2010.

[19] R. M. Silverstein, F. X. Webster, and D. J. Kiemle, Spectrometric Identification of Organic Compounds, John Wiley \& Sons, Princeton, NJ, USA, 2005.

[20] A. Kaniyoor and S. Ramaprabhu, "Enhanced efficiency in dye sensitized solar cells with nanostructured Pt decorated multiwalled carbon nanotube based counter electrode," Electrochimica Acta, vol. 72, pp. 199-206, 2012.

[21] J. Zhang, H. Zou, Q. Qing et al., "Effect of chemical oxidation on the structure of single-walled carbon nanotubes," Journal of Physical Chemistry B, vol. 107, no. 16, pp. 3712-3718, 2003.

[22] K. A. Wepasnick, B. A. Smith, J. L. Bitter, and D. H. Fairbrother, "Chemical and structural characterization of carbon nanotube surfaces," Analytical and Bioanalytical Chemistry, vol. 396, no. 3, pp. 1003-1014, 2010.

[23] Y. Xing, L. Li, C. C. Chusuei, and R. V. Hull, "Sonochemical oxidation of multiwalled carbon nanotubes," Langmuir, vol. 21, no. 9, pp. 4185-4190, 2005.

[24] J. Hilding, E. A. Grulke, Z. G. Zhang, and F. Lockwood, "Dispersion of carbon nanotubes in liquids," Journal of Dispersion Science and Technology, vol. 24, no. 1, pp. 1-41, 2003.

[25] M. S. Dresselhaus, G. Dresselhaus, R. Saito, and A. Jorio, "Raman spectroscopy of carbon nanotubes," Physics Reports, vol. 409, no. 2, pp. 47-99, 2005.

[26] R. Graupner, "Raman spectroscopy of covalently functionalized single-wall carbon nanotubes," Journal of Raman Spectroscopy, vol. 38, no. 6, pp. 673-683, 2007.

[27] X. Zhang, L. Lei, B. Xia, Y. Zhang, and J. Fu, "Oxidization of carbon nanotubes through hydroxyl radical induced by pulsed $\mathrm{O}_{2}$ plasma and its application for $\mathrm{O} 2$ reduction in electroFenton," Electrochimica Acta, vol. 54, no. 10, pp. 2810-2817, 2009.

[28] K. Imasaka, J. Suehiro, Y. Kanatake, Y. Kato, and M. Hara, "Preparation of water-soluble carbon nanotubes using a pulsed streamer discharge in water," Nanotechnology, vol. 17, no. 14, pp. 3421-3427, 2006.

[29] D. Kolacyak, J. Ihde, C. Merten, A. Hartwig, and U. Lommatzsch, "Fast functionalization of multi-walled carbon nanotubes by an atmospheric pressure plasma jet," Journal of Colloid and Interface Science, vol. 359, no. 1, pp. 311-317, 2011.

[30] O. Matarredona, H. Rhoads, Z. Li, J. H. Harwell, L. Balzano, and D. E. Resasco, "Dispersion of single-walled carbon nanotubes in aqueous solutions of the anionic surfactant NaDDBS," The Journal of Physical Chemistry B, vol. 107, no. 48, pp. 13357-13367, 2003.

[31] J. Yu, N. Grossiord, C. E. Koning, and J. Loos, "Controlling the dispersion of multi-wall carbon nanotubes in aqueous surfactant solution," Carbon, vol. 45, no. 3, pp. 618-623, 2007. 

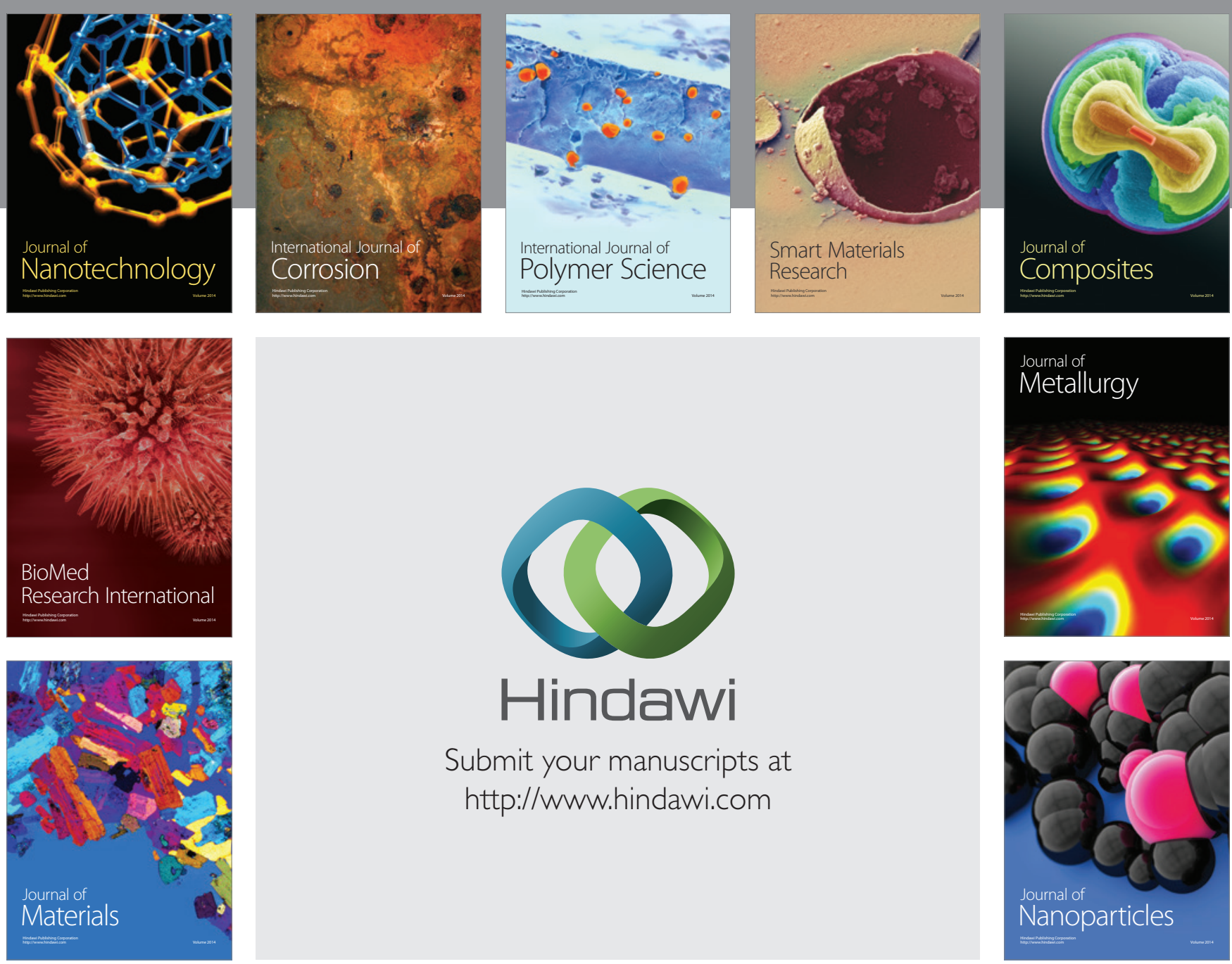

Submit your manuscripts at http://www.hindawi.com
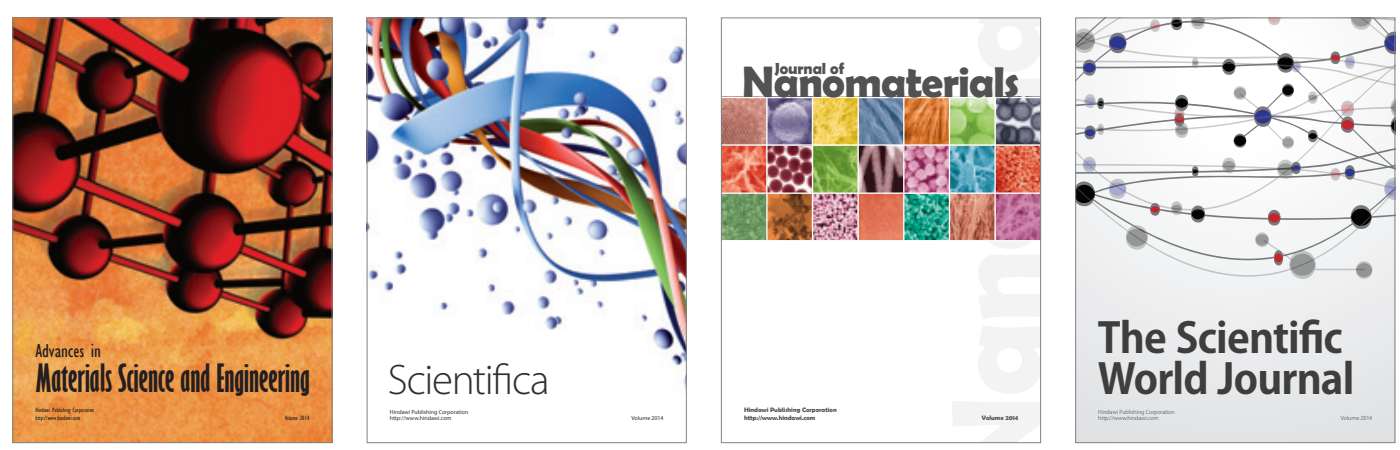

\section{The Scientific World Journal}
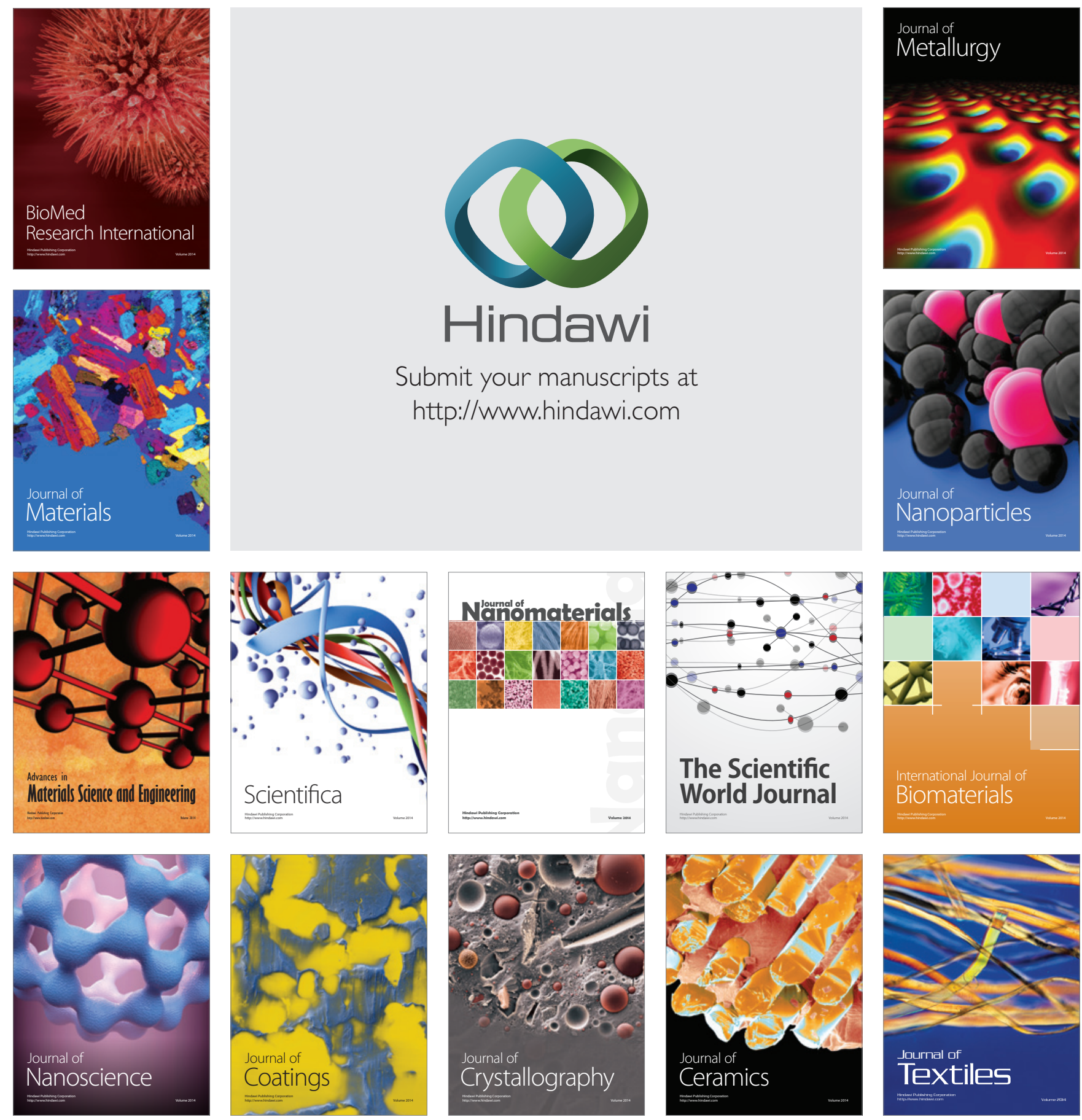